\title{
Dynamics of High-Energy Multimode Raman Solitons
}

\author{
M. Ferraro ${ }^{1}$, M. Zitelli ${ }^{1}$, F. Mangini' ${ }^{2}$, D. S. Kharenko ${ }^{3}$, A. Niang $^{2}$, and S. Wabnitz ${ }^{1,3}$ \\ ${ }^{1}$ Department of Information Engineering, Electronics and Telecommunications (DIET), Sapienza University of \\ Rome, Via Eudossiana 18, 00184 Rome, Italy \\ ${ }^{2}$ Department of Information Engineering (DII), University of Brescia, Via Branze 38, 25123 Brescia, Italy \\ ${ }^{3}$ Novosibirsk State University, Pirogova 1, Novosibirsk 630090, Russia
}

\begin{abstract}
The dynamics of high-energy Raman solitons in graded-index multimode fibers is both numerically and experimentally investigated. The propagation of high-power pulses produces nonlinear losses, that quench up to $80 \%$ of the fiber transmission. In such a regime, several solitons arising from the fission of ultra-short femtosecond pulses manifest unique features: pulse width, Raman self-frequency shift and soliton order remain nearly constant over a broad range of energies.
\end{abstract}

Keywords: optical solitons, optical fibers, Kerr effect, Raman scattering, ultrashort phenomena.

\section{INTRODUCTION}

The study of nonlinear optical effects in multimode (MM) optical fibers dates back to the ' 80 s. However, the manipulation of the temporal and spectral properties of ultrashort pulses, combined with the degrees of freedom provided by fiber multimodality is a research field that has only emerged over the last few years [1]. As a matter of fact, MM fibers hold a promise to increase the capacity of next-generation communications systems, and to scale up the energy of supercontinuum and fiber laser sources. Still, complex pulse propagation phenomena in MM fibers are not yet fully understood. In particular, MM solitons, that result from the simultaneous compensation, by the Kerr effect, of chromatic dispersion and modal dispersion, have not yet even nearly been investigated as widely as their single mode (SM) counterparts. The Raman-induced MM soliton self-frequency shift (SSFS) in graded-index (GRIN) MM fibers was studied in 2013 in a low pulse energy (up to $3 \mathrm{~nJ}$ ) regime: there, MM solitons were mostly carried by the fundamental mode of the MM fiber [2]. The more general spatiotemporal behavior of MM solitons, including their formation and fission, was later explored by Wright et al., in a regime where the input energy distribution involves the contribution of several guided modes [3-5]. Raman MM solitons at $2100 \mathrm{~nm}$ could be generated from a GRIN fiber, by using $500 \mathrm{fs}$ input pump pulses at $1550 \mathrm{~nm}$ with energies up to $21 \mathrm{~nJ}$.

In this work, we study the high-energy (up to $550 \mathrm{~nJ}$, limited by the damage threshold of the fiber) regime of femtosecond pulse propagation in a standard parabolic GRIN MM fiber with anomalous dispersion. We reveal a new optical pulse propagation regime associated with the fission of the input multi-soliton pulse, leading to decay into several individual fundamental MM solitons. The MM solitons are first generated at a set of different wavelengths (or wavelength multiplex), before undergoing SSFS. Remarkably, these MM solitons exhibit nearly the same temporal duration, in spite of their different wavelengths and associated dispersions. Soliton propagation in GRIN MM fibers can be approximated by a simple single mode (SM) model, involving a spatially varying effective mode area [6]. Our experiments show that such an SM soliton approximation is indeed valid, but only over a limited range of soliton energies. Moreover, we also reveal several unexpected properties of high-energy pulse propagation in MM GRIN fibers. In particular, the presence of a nonlinear loss mechanism, leading to output energy clamping and SSFS suppression. Such losses are due to multiphoton absorption by the defects of the Germanium doped fiber [7], leading to blue luminescence that is side-scattered from the fiber, at points where the self-imaging shrinks the beam waist [8]. Nonlinear losses quench up to $80 \%$ of the transmission, already from the first few centimetres of fiber.

\section{EXPERIMENTAL SETUP}

We carried out our experiments in the anomalous dispersion regime of a standard GRIN fiber. We used laser pulses with hyperbolic secant temporal profile of $130 \mathrm{fs}$ duration at $1550 \mathrm{~nm}$. At this wavelength, the estimated dispersion parameters up to the fourth order read as: $\beta_{2}=-22 \mathrm{ps}^{2} / \mathrm{km}, \beta_{3}=0.132 \mathrm{ps}^{3} / \mathrm{km}$ and $\beta_{4}=-5 \times 10^{-4} \mathrm{ps}^{4} / \mathrm{km}$, while the nonlinear coefficient $n_{2}=2.7 \times 10^{-20} \mathrm{~m}^{2} / \mathrm{W}$. Our laser system involves a hybrid optical parametric amplifier (OPA) of white-light continuum (Light Conversion ORPHEUS-F), pumped by a femtosecond Ybbased laser (Light Conversion PHAROS-SP-HP), generating pulses with $25-100 \mathrm{kHz}$ repetition rate. The input pulse energy was varied between $0.1 \mathrm{~nJ}$ and $500 \mathrm{~nJ}$ with a variable external attenuator.

In our experiments, the pulse is focused into a $30 \mathrm{~cm}$ long GRIN MM fiber. The beam diameter at the fiber input is $20 \mu \mathrm{m}$, while the core and cladding radii are respectively 25 and $62.5 \mu \mathrm{m}$. The cladding index is $n_{\text {clad }}=1.457$ while the relative core-cladding index difference $\Delta=0.0103$. 


\section{RAMAN SOLITON FISSION}

As shown by the measured output spectra in Fig. 1a, fission of the input, multi-soliton pulse leads to the creation of several, wavelength-shifted Raman solitons at the fiber output. In particular, one can observe from Fig. 1a how the Raman-induced SSFS tends to saturate to a fixed value for energies higher than $250 \mathrm{~nJ}$. Correspondingly, the total output energy (both spectrally filtered above $1100 \mathrm{~nm}$ and measured across all bandwidth) remains clamped, as Fig. 1b shows.

These observations allow us to infer the presence of nonlinear losses. We can ascribe them to multiphoton absorption phenomena, as well as to light scattering outside the fiber, which is particularly relevant at those points where the beam shrinks due to self-imaging, leading to local peaks of light intensity. We can therefore define the existence of two separated nonlinear propagation regimes: a first one ("linear loss" regime), where the output energy linearly grows with the input energy, and a second one, where the output energy is clamped to a nearly constant value, dubbed "nonlinear loss" regime. From Fig. 1b, we can set at approximately $100 \mathrm{~nJ}$ the input beam energy boundary between these two regimes. For input energies higher than $500 \mathrm{~nJ}$, we observed irreversible fiber damage.

(a)

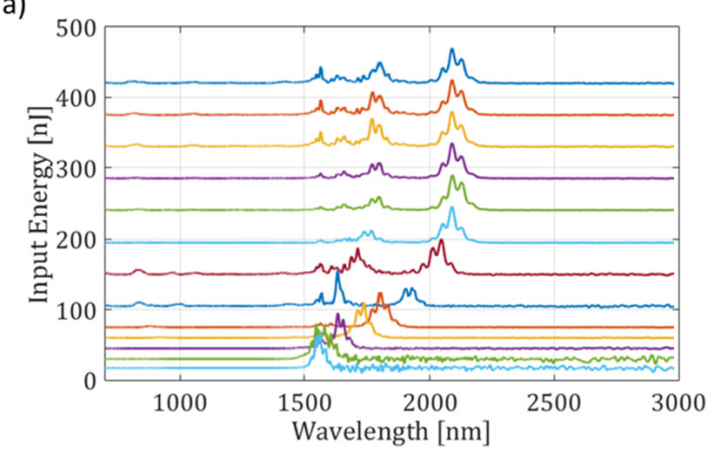

(b)

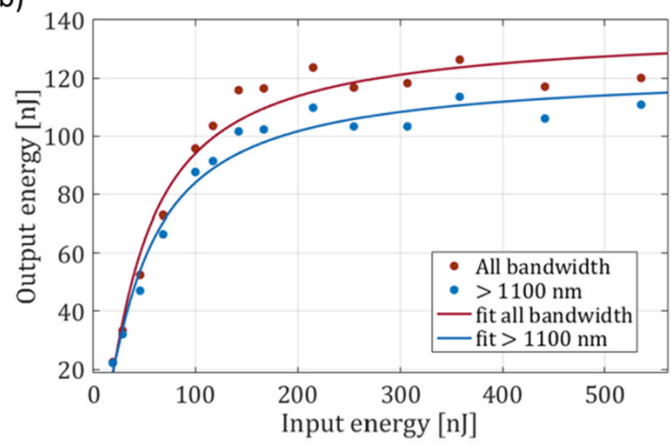

Figure 1: (a) Output spectral intensity (linear scale) at different input energies from $30 \mathrm{~cm}$ of GRIN MM fiber; (b) Experimental output energy vs. input energy, in the overall bandwidth and for wavelengths above $1100 \mathrm{~nm}$.

In order to better understand the dynamics of Raman soliton formation, we performed a cut-back experiment. In Fig. 2a we report how the spectrum evolves upon propagation. The figure shows how the input pulse undergoing the fission process results in the generation of a wavelength multiplex of different Raman solitons after about $7 \mathrm{~cm}$ of propagation. In Fig. 2a one can also observe the generation of anti-Stokes dispersive wave sidebands, originating from spatiotemporal MM soliton oscillations [5].

Interestingly, this experiment also evidences how the transmission losses from the GRIN MM fiber are strongly dependent on its length. From Fig. $2 b$ one can see how the normalized transmitted energy remains nearly constant after the first centimeters of propagation, both in the "linear loss" and in the "nonlinear loss" regime. On the other hand, the fiber transmission is quenched at the very beginning of the fiber, dropping down to only $20 \%$ for $400 \mathrm{~nJ}$ of input energy.

(a)

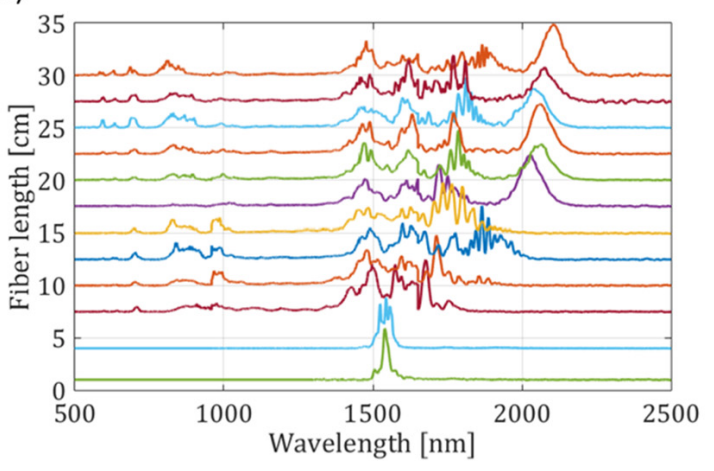

(b)

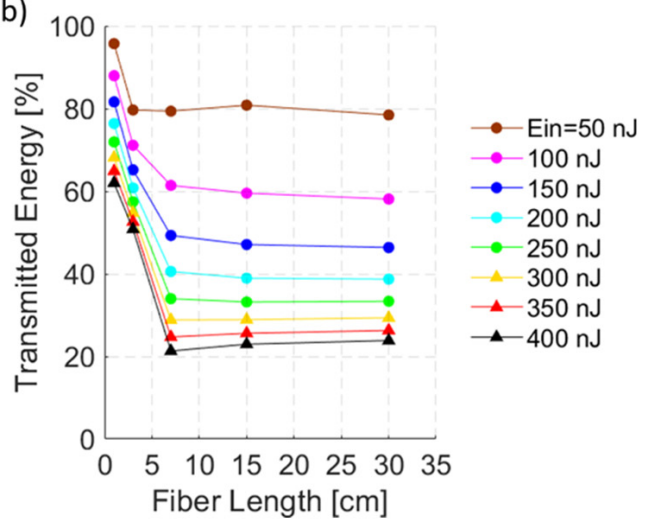

Figure 2: (a) Output spectrum evolution at different fiber lengths. The input energy is 300 nJ; (b) Normalized transmitted energy vs. fiber length for different input energies.

\section{NUMERICAL SIMULATIONS}

The fission process could be reproduced by numerical simulations, using an exact $3 \mathrm{D}+1$ vectorial model including second, third, and fourth order dispersion, Kerr and Raman nonlinearities. We neglect the contribution 
of polarization mode dispersion, since it is negligible for the fiber lengths involved in our experiments. We include a phenomenological two-photon absorption (TPA) term, in order to take into account of the presence of nonlinear losses: the TPA coefficient is taken to be $\alpha_{2}=10^{-16} \mathrm{~m} / \mathrm{W}$ by fitting the experimental SSFS values. Random phase noise was added to the input field, in order to describe the generation of intensity speckles in the output transverse field, and to seed the generated dispersive waves. Random changes in the fiber core diameter have also been introduced, although they produced negligible effects over the considered fiber lengths.

Numerical simulations could only be performed for input energies in the "linear loss" regime, below $110 \mathrm{~nJ}$. Beyond this threshold, simulations cannot reach the fiber output end, owing to beam filamentation that occurs over the first few centimetres of propagation. In contrast, we only experimentally observed fiber damage for input energies above $550 \mathrm{~nJ}$. The discrepancy between simulated and experimental threshold for catastrophic self-focusing can be ascribed to excess multiphoton absorption [7], and to energy side-scattering processes [8], both leading to significant additional losses, which saturate the beam intensity and prevent is collapse. Such mechanisms are not yet incorporated in our model, which only includes TPA.

(a)

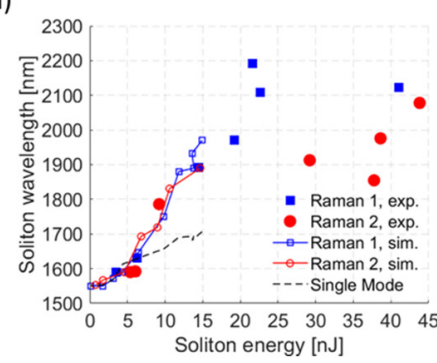

(b)

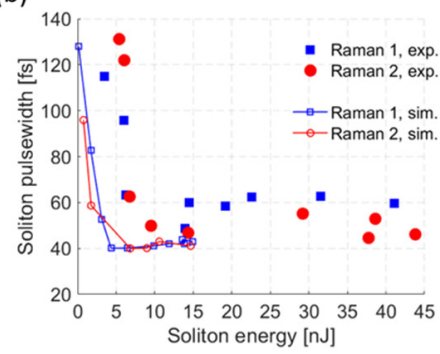

(c)

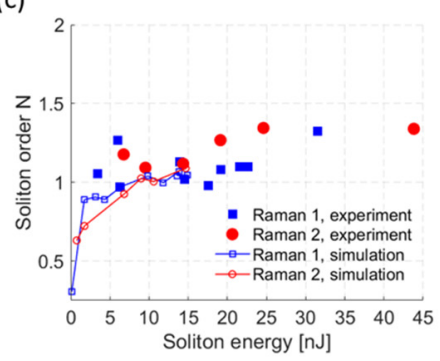

Figure 3. Output Raman soliton wavelength, pulse width and order $(a, b, c)$ vs. soliton energy.

\section{MULTIMODE SOLITON DYNAMICS}

By comparing simulations to experiments for input pulse energies in the "linear loss" regime, we have studied the dynamics of the generated MM Raman solitons as a function of their energy. Remarkably, at relatively low soliton energies our numerical simulations permit to reproduce well the experimental values for the soliton wavelengths. As can be seen in Fig. 3a, for energies up to $15 \mathrm{~nJ}$ a good agreement is obtained between theory and experiments for the wavelengths of the most red-shifted solitons, labelled Raman 1 and 2, respectively. On the other hand, for energies above $15 \mathrm{~nJ}$, which corresponds to propagation in the "nonlinear loss regime", the Raman soliton wavelengths exhibit a chaotic distribution, fluctuating around a constant value, as shown by the spectra reported in Fig. 1a. In Fig. 3a we also compare the simulated SSFS with the value that is predicted by an SM theory. The latter predicts a linear dependence on soliton energy. As the figure shows, the SM approximation only works for energies below $5 \mathrm{~nJ}$.

Another prediction of numerical simulations is the generation of a soliton wavelength multiplex with nearly equal pulse widths. This is also confirmed by our experimental analysis of the temporal duration of the different Raman solitons, that are generated by the fission process. In Fig. $3 \mathrm{~b}$ one can remark how both simulations and the experiments show that the soliton pulse width remains nearly constant as the soliton energy is increased. The simulations are able to catch such behavior whenever pulse propagation occurs in the "linear loss" regime, that is before the occurrence of beam collapse. Experimentally, we confirmed that solitons forming the multiplex indeed maintain a constant temporal duration of about $50-60 \mathrm{fs}$, even in the "nonlinear loss" regime (that is, for soliton energies above $15 \mathrm{~nJ}$ ).

By comparing the results shown in Fig. $3 a$ and $3 b$, we can state that the wavelength multiplex self-organizes in solitons with equal pulse widths and unequal amplitudes. Such a configuration corresponds to a regime with minimal emission of dispersive waves (or radiation), since nonlinearity exactly balances the local dispersion (as it is determined by second and third-order dispersion) at each of the different Raman wavelengths [9].

Our model predicts that the solitons produced by the fission are fundamental MM solitons. In Fig. 3c we report the output soliton order $\mathrm{N}$ as a function of its energy. Here the soliton order is calculated by the SM formula [6]

$$
N^{2}=\frac{n_{2} T_{0} E}{\lambda\left|\beta_{2}\right| w}
$$

where $w, \lambda, E$ and $T_{0}$ are the soliton beam waist, wavelength, energy and pulse width, respectively. We assume the beam waist to be the same as that of the input pulse, for all wavelengths and energies. Figure $3 \mathrm{c}$ shows that the solitons keep the same order $N$ which remains close to unity, nearly independently of their energies. In the "nonlinear loss" regime, i.e., for $E>15 \mathrm{~nJ}$, experimental soliton orders turn out to be always higher than unity, with values ranging between 1 and 1.35. This may be associated with the MM nature of the solitons, that require 
a higher energy with respect to SM solitons, in order to compensate, via nonlinearity, for modal dispersion (i.e., nonlinearly trap the fiber modes together in time), in addition to chromatic dispersion.

\section{CONCLUSIONS}

We experimentally and numerically investigated the dynamics of high-energy multimode Raman solitons in a parabolic GRIN MM fiber. We showed that a high-energy pulse propagating inside the fiber undergoes a fission process, producing a set of wavelength-shifted Raman solitons. These solitons spread apart across the spectral domain under the action of SSFS, and consist of MM fundamental solitons with nearly equal temporal durations, and different energies. For input pulse energies above $100 \mathrm{~nJ}$, and hundreds of $\mathrm{kW}$ of peak power, nonlinear losses due to multiphoton absorption and associated side-scattering of blue photo-luminescence were experimentally observed. Nonlinear losses dramatically limit the transmission of the input pulse energy after the first centimetres of propagation along fiber. Raman solitons generated at different wavelengths have, independently of their energy, nearly the same pulse width (about 50-60 fs). In the same way, the SSFS is also clamped to a constant value. The generation of soliton wavelength multiplexes with equal pulse widths and unequal amplitudes minimizes the emission of radiative waves, since both second and third order dispersion are balanced by nonlinearity at each soliton wavelength. Our findings provide a new insight in the dynamics of MM GRIN fiber solitons, and may be relevant for the development of novel fiber based sources of high-energy ultrashort pulses in the near and mid-infrared.

\section{ACKNOWLEDGEMENTS}

We acknowledge support from the European Research Council (ERC) under the European Union's Horizon 2020 research and innovation program (grant No. 740355), and by the Russian Ministry of Science and Education, Grant No 14.Y26.31.0017.

\section{REFERENCES}

[1] K. Krupa et al.: Multimode nonlinear fiber optics, a spatiotemporal avenue, APL Photonics, vol. 11, p. 110901, 2019.

[2] W. H. Renninger et al.: Optical solitons in graded-index multimode fibres, Nat. Commun., vol. 4, p. 1719, 2013.

[3] L. G. Wright et al.: Spatiotemporal dynamics of multimode optical solitons, Opt Express, vol. 23, p. 3492, 2015.

[4] L. G. Wright et al.: Controllable spatiotemporal nonlinear effects in multimode fibres, Nat. Photonics, vol. 9, p. 306, 2015.

[5] L. G. Wright et al.: Ultrabroadband dispersive radiation by spatiotemporal oscillation of multimode waves, Phys. Rev. Lett., vol. 115, p. 223902, 2015.

[6] A. S. Ashan and G. P. Agrawal: Spatio-temporal enhancement of Raman-induced frequency shift in graded index multimode fibers, Opt. Lett., vol. 44, p. 2637, 2019.

[7] P.G. Kazansky et al.: Anomalous anisotropic light scattering in Ge-doped silica glass. Phys. Rev. Lett., vol. 82, p. 2199, 1999.

[8] T. Hansson et al:: Nonlinear beam self-imaging and self-focusing dynamics in a GRIN multimode optical fiber: theory and experiments, 2020, https://arxiv.org/abs/2005.07280v1.

[9] Y. Kodama, A. V. Mikhailov, and S. Wabnitz: Input pulse optimization in wavelength-divisionmultiplexed soliton transmissions, Opt. Commun., vol. 143, pp. 53-56, 1997. 